\title{
Novel Porphyrin-Fullerene Assemblies: from Rotaxanes to Catenanes
}

David I. Schuster, ${ }^{* a}$ Ke Li, ${ }^{a}$ Dirk M. Guldi, ${ }^{* b}$ and Jeff Ramey ${ }^{b}$

${ }^{a}$ Chemistry Department, New York University, New York City, NY 10003, USA;

${ }^{b}$ Radiation Laboratory, University of Notre Dame, Notre Dame, IN 46556, USA;

Email Addresses: david.schuster@nyu.edu; guldi.1@nd.edu. 


\section{Synthesis of Macrocycle 4.}

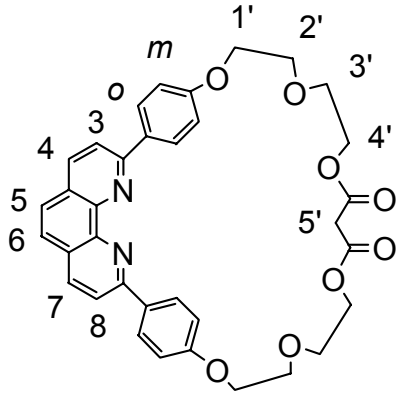

4

Freshly distilled malonyl dichloride (350 $\mathrm{mg}, 2.5 \mathrm{mmol})$ in dry $\mathrm{CH}_{2} \mathrm{Cl}_{2}(50 \mathrm{~mL})$ was added dropwise to a mixture of $\mathbf{3}(1.35 \mathrm{~g}$, $2.5 \mathrm{mmol})$, triethylamine $(0.5 \mathrm{~mL})$ and DMAP $(10 \mathrm{mg})$ in dry $\mathrm{CH}_{2} \mathrm{Cl}_{2}(300 \mathrm{~mL})$ at $0{ }^{\circ} \mathrm{C}$ in a 1 hour period. Then the yellow solution was stirred at $0{ }^{\circ} \mathrm{C}$ for 5 hours then at room temperature for 10 hours. The solution was extracted with water and brine, dried over anhydrous $\mathrm{MgSO}_{4}$ and the solvent was removed in vacuo. The yellow oil was purified by flash chromatography. ( $\mathrm{SiO}_{2}, \mathrm{CH}_{2} \mathrm{Cl}_{2}: \mathrm{MeOH}=97: 3$ ). Light yellow solid was obtained from the first fraction. (200mg, 13\%)

${ }^{1} \mathrm{H}$ NMR $\left(200 \mathrm{MHz}, \mathrm{CDCl}_{3}\right) \delta 8.45(\mathrm{~d}, 4 \mathrm{H}, \mathrm{Ho}), 8.26\left(\mathrm{~d}, 2 \mathrm{H}, \mathrm{H}_{4,7}\right), 8.08\left(\mathrm{~d}, 2 \mathrm{H}, \mathrm{H}_{3,8}\right) 7.76$ $\left(\mathrm{s}, 2 \mathrm{H}, \mathrm{H}_{5,6}\right), 7.14(\mathrm{~d}, 4 \mathrm{H}, \mathrm{Hm}), 4.4-4.2\left(\mathrm{~m}, 8 \mathrm{H}, \mathrm{H}_{1}, 4^{\prime}\right), 3.9-3.7\left(\mathrm{~m}, 8 \mathrm{H}, \mathrm{H}_{2}, 3^{\prime}\right), 3.54$ (s, $2 \mathrm{H}, \mathrm{H}_{5}$, )

ESI-MS: cal.608.2, found $609.1[\mathrm{M}+\mathrm{H}]^{+}$.

\section{Synthesis of fullerenomacrocycle 5 .}

Macrocycle 4 (43mg, $0.07 \mathrm{mmmol}), \mathrm{C}_{60}$ (80mg, $0.11 \mathrm{mmol}$ ) and $\mathrm{CBr}_{4}(40 \mathrm{mg}, 0.12$ $\mathrm{mmol})$ were dissolved in dry toluene $(80 \mathrm{~mL})$ under $\mathrm{N}_{2}$ atmosphere. DBU $(40 \mathrm{mg}, 0.26$ $\mathrm{mmol}$ ) was added to the above solution at room temperature and the solution was stirred at room temperature for 10 hours. The mixture was purified by flash column chromatography. $\left(\mathrm{SiO}_{2}\right.$, toluene: $\left.\mathrm{MeOH}=97: 3\right)$. The first band was unreacted $\mathrm{C}_{60}$, the second band is the ideal product $\mathbf{5}$. (brown solid, $48 \mathrm{mg}, 52 \%$ )

${ }^{1} \mathrm{H}$ NMR (200 MHz, $\left.\mathrm{CDCl}_{3}\right) \delta 8.37$ (d, 4H, Ho), 8.20 (d, 2H, $\left.\mathrm{H}_{4,7}\right), 8.02\left(\mathrm{~d}, 2 \mathrm{H}, \mathrm{H}_{3,8}\right)$, $7.70\left(\mathrm{~s}, 2 \mathrm{H}, \mathrm{H}_{5,6}\right), 7.10(\mathrm{~d}, 4 \mathrm{H}, \mathrm{Hm}), 4.72\left(\mathrm{t}, 4 \mathrm{H}, \mathrm{H}_{4}\right), 4.22\left(\mathrm{t}, 4 \mathrm{H}, \mathrm{H}_{1}\right.$ ) $), 3.9-3.7(\mathrm{~m}, 8 \mathrm{H}$, $\left.\mathrm{H}_{2}, 3^{\prime}\right)$.

FAB-MS: cal. 1326.8, found $1328.2[\mathrm{M}+\mathrm{H}]^{+}$

\section{Synthesis of linear thread 6.}

Porphyrin $\operatorname{acid}^{1}$ (750mg, $\left.0.75 \mathrm{mmol}\right), 3$ (810 $\left.\mathrm{mg}, 1.5 \mathrm{mmol}\right)$, EDC (285mg, $\left.1.5 \mathrm{mmol}\right)$, and DMAP (10mg, $0.08 \mathrm{mmol}$ ) were place in a $250 \mathrm{~mL}$ round bottom flask under $\mathrm{N}_{2}$ atmosphere. Dry $\mathrm{CH}_{2} \mathrm{Cl}_{2} 150 \mathrm{~mL}$ was added under $\mathrm{N}_{2}$. The purple mixture was stirred at room temperature for 12 hours. The solution was extracted with dilute $\mathrm{HCl}$ followed by water and dried over anhydrous sodium sulfate. The solvent was removed under reduced pressure. The crude product was purified by flash chromatography. $\left(\mathrm{SiO}_{2}, \mathrm{CH}_{2} \mathrm{Cl}_{2}\right.$ : $\mathrm{MeOH}=97: 3$ ) (purple solid, 960mg, 85\%). The purple product $(300 \mathrm{mg}, 0.2 \mathrm{mmol}$ ) was dissolved in chloroform $(50 \mathrm{~mL})$ and zinc acetate $(150 \mathrm{mg})$ in methanol $(5 \mathrm{~mL})$ was added. The mixture was refluxed for 1 hour. The purple solution was extracted with water and dried over anhydrous sodium sulfate. The solvent was removed under reduced

${ }^{1}$ Luo, C.; Guldi, D. M.; Imahori, H.; Tamaki, K.; Sakata, Y. J. Am. Chem. Soc, 2000, 122, 6535 
pressure. The crude product was purified by pass though a short silica column. $\left(\mathrm{CH}_{2} \mathrm{Cl}_{2}\right.$ : $\mathrm{MeOH}=97: 3)$.

${ }^{1} \mathrm{H}$ NMR $\left(400 \mathrm{MHz}, \mathrm{CDCl}_{3}\right) \delta 9.02(\mathrm{~s}, 4 \mathrm{H}), 8.89(\mathrm{~s}, 2 \mathrm{H}, J=7 \mathrm{~Hz}), 8.75(\mathrm{~d}, 2 \mathrm{H}, J=7$ $\mathrm{Hz}), 8.42-8.34(\mathrm{~m}, 6 \mathrm{H}), 8.25(\mathrm{~d}, 2 \mathrm{H}, J=9 \mathrm{~Hz}), 8.12-8.10(\mathrm{~m}, 6 \mathrm{H}), 7.87(\mathrm{~d}, 1 \mathrm{H}, J=1.6$ $\mathrm{Hz}), 7.79-7.78(\mathrm{~m}, 3 \mathrm{H}), 7.73(\mathrm{~d}, 1 \mathrm{H}, J=8 \mathrm{~Hz}), 7.62(\mathrm{~d}, 1 \mathrm{H}, J=8 \mathrm{~Hz}), 7.50(\mathrm{~d}, 1 \mathrm{H}, J=$ $1.6 \mathrm{~Hz}), 7.18(\mathrm{~d}, 2 \mathrm{H}, J=9 \mathrm{~Hz}), 7.08(\mathrm{~d}, 1 \mathrm{H}, J=8 \mathrm{~Hz}), 6.93(\mathrm{~d}, 1 \mathrm{H}, J=8 \mathrm{~Hz}), 6.90(\mathrm{~d}$, $2 \mathrm{H}, J=9 \mathrm{~Hz}), 4.68(\mathrm{t}, 2 \mathrm{H}), 4.37(\mathrm{t}, 2 \mathrm{H}), 4.06(\mathrm{~m}, 4 \mathrm{H}), 3.92(\mathrm{t}, 2 \mathrm{H}), 3.39(\mathrm{t}, 2 \mathrm{H}), 2.81(\mathrm{t}$, $2 \mathrm{H}), 2.52(\mathrm{~b}, 2 \mathrm{H}), 1.52(\mathrm{~m}, 54 \mathrm{H})$.

MALDI-TOF Mass: cal. 1578.8 , found $1579.6[\mathrm{M}+\mathrm{H}]^{+}$ 


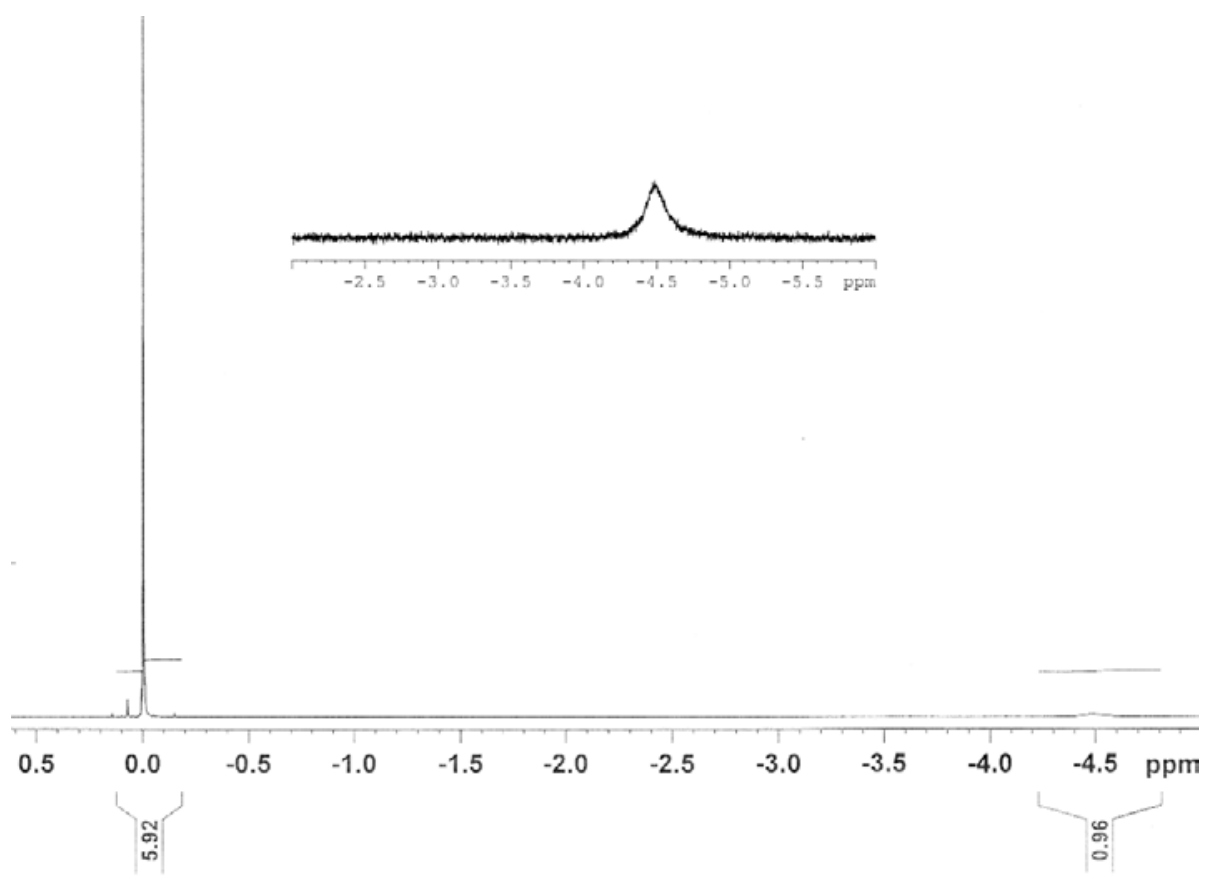

NMR (400 MHz, 298K, $\mathrm{CDCl}_{3}$ ) DABCO: rotaxane $\mathbf{2}=1: 1$

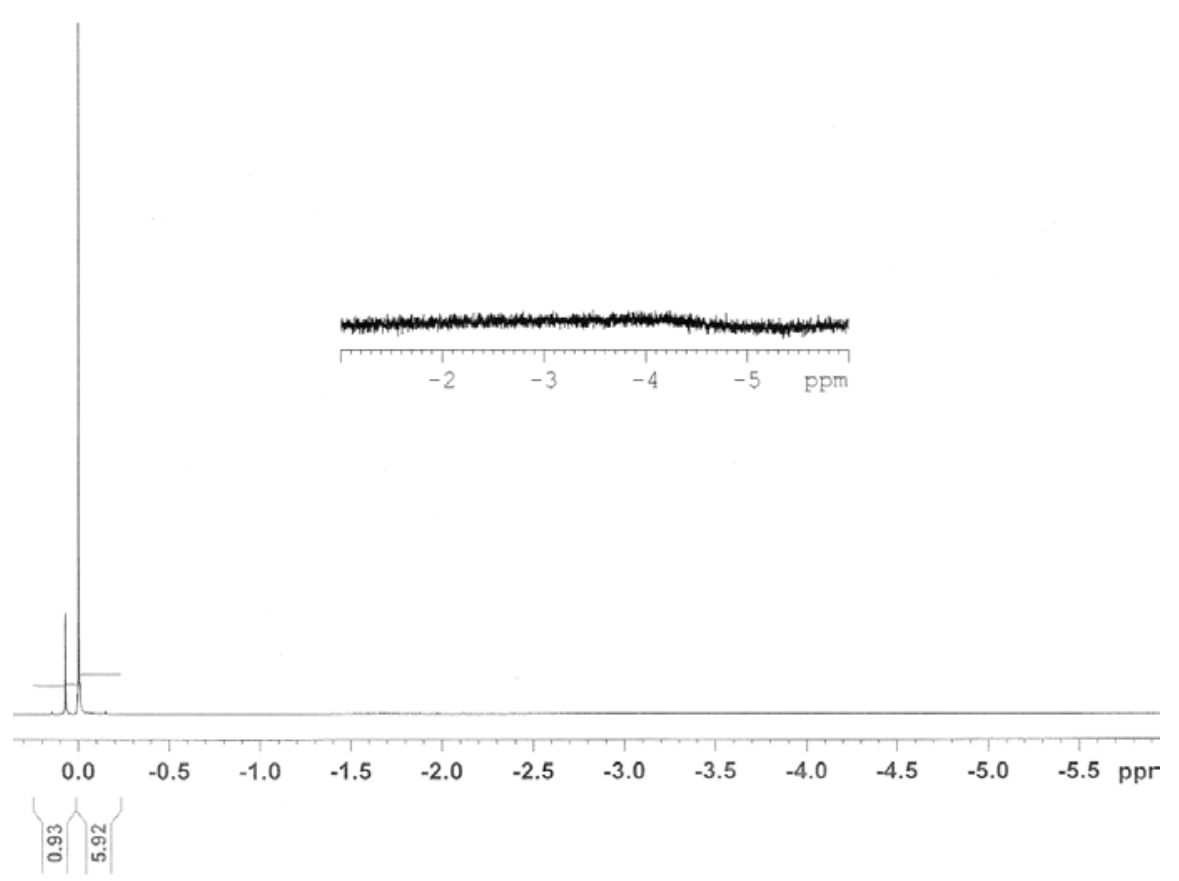

NMR (400 MHz, 298K, $\mathrm{CDCl}_{3}$ ) DABCO: rotaxane 2 = 2:1 